ASCLEPIO. Revista de Historia de la Medicina y de la Ciencia

67 (2), julio-diciembre 2015, p107

ISSN-L:0210-4466

http://dx.doi.org/10.3989/asclepio.2015.25

DOSSIER: EL NACIMIENTO DE LA PSIQUIATRÍA: UN MOVIMIENTO EUROPEO /

THE BIRTH OF PSYCHIATRY: A EUROPEAN MOVEMENT

\title{
LA PASIÓN DE ALEXANDER CRICHTON
}

\author{
Daniel Matusevich \\ Hospital Italiano de Buenos Aires (HIBA)
}

Recibido: 15 de mayo de 2015; Aceptado: 1 de septiembre de 2015.

Cómo citar este artículo/Citation: Matusevich, Daniel (2015), "La pasión de Alexander Crichton", Asclepio, 67 (2): p107. doi: http:// dx.doi.org/10.3989/asclepio.2015.25

RESUMEN: Alexander Crichton, nació en Edimburgo en 1763, se graduó en la Universidad de Leyden y completó su formación médica en Berlín, Paris, Stuttgart, Praga y Gotinga, vinculándose estrechamente con el ambiente social y cultural de esos prestigiosos centros europeos. En el año 1798 publicó An Inquiry into the nature and origin of Mental Derangement (Investigación sobre la naturaleza y el origen del trastorno mental), única obra de su autoría dedicada a la locura. En este artículo se analizan algunas cuestiones referidas al método general que aplica el autor para la concepción de su obra, sus contribuciones a la semiología de la mente, al análisis del proceso de envejecimiento y a la función de la atención, para detenernos, finalmente, en su conceptuación del mundo de las pasiones y de la locura que fueron retomadas por otros autores como Philippe Pinel y Dominique Esquirol, y que constituyeron un antecedente fundamental en el nacimiento de la psiquiatría.

PALABRAS CLAVE: Alexander Crichton; Philippe Pinel; Dominique Esquirol; Pasiones; Historia de la psiquiatría.

\section{ALEXANDER CRICHTON'S PASSION}

ABSTRACT: Alexander Crichton was born in Edinburgh in 1763; he graduated from Leyden University and travelled through Berlín, Paris, Stuttgart, Prague and Gotinga to perfect his knowledge in medicine while he establish a deep connection between the social media and the culture of those places. On 1798 he published An inquiry into the nature and origin of Mental Derangement, the only book he wrote dedicated to madness. In this paper we review some questions referred to the general method that the author applies, his contributions to the semiotics of the mind and to the analysis of the process of aging and the function of the attention; we also take a look to his conception of the world of passions and of madness, who influenced Philippe Pinel and Dominique Esquirol and also constitute a cornerstone in the birth of psychiatry.

KEY WORDS: Alexander Crichton; Philippe Pinel; Dominique Esquirol; Passions; Psychiatry History.

Copyright: () 2015 CSIC. Este es un artículo de acceso abierto distribuido bajo los términos de la licencia Creative Commons Attribution-Non Commercial (by-nc) Spain 3.0. 


\section{APUNTES BIOGRÁFICOS}

Alexander Crichton nació en Edimburgo en 1763, cursó sus estudios de medicina en la universidad de Leiden, en la que fue discípulo de los cirujanos Alexander Wood ${ }^{1}$ y William Fordyce ${ }^{2}$, y se graduó en 1785. Crichton pasó el invierno de 1785-1786 en Paris adonde presumiblemente conoció a Pinel según relata en $1842^{3}$ (Weiner, 1999). Durante los tres años siguientes perfeccionó su formación en Alemania. Esa etapa de sus estudios lo marco de manera indeleble y condicionó sus producciones científicas posteriores. Durante el verano de 1786 estudió alemán en Stuttgart, luego pasó el invierno en Viena siguiendo las enseñanzas de Maximilian Stoll ${ }^{4}$, y en Halle, adonde vivió con la familia de su maestro Phillipp Meckel $^{5}$, para después trasladarse durante casi un año a Berlín. Finalmente, como culminación de su periplo germano, pasó los últimos seis meses en Gotinga formando parte del círculo de discípulos de Johann Friedrich Blumenbach $(1752-1840)^{6}$ (Tansey, 1983; Weiner, 1990; Appleby, 1999, 2004; Stagnaro, 2013).

Weiner sostiene que la influencia de Kant, cuya filosofía conoció Crichton durante su estancia en Alemania, en relación a la oposición entre el mundo de la racionalidad y el de las pasiones definió la perspectiva de Crichton en referencia a la locura (Weiner, 1990).

En 1792, de regreso en Inglaterra, Crichton tradujo al idioma inglés el ensayo de Blumenbach "An Essay on Generation», libro de fisiología que lo inspiró para escribir, en 1798, An Inquiry into the nature and origin of Mental Derangement («Investigación sobre la naturaleza y el origen del trastorno mental»), posteriormente traducido al holandés, alemán y francés (Stagnaro, 2013).

En 1794, Crichton comenzó a trabajar en el hospital de Westminster como médico cirujano, donde también se dedicó a la enseñanza de la medicina y la química. En mayo de 1800, al ser elegido miembro de la Royal Society, dejó su puesto en el hospital y, aproximadamente para la misma fecha, fue designado médico del duque de Cambridge.

Entre 1804 y 1819 desarrolló su práctica en Rusia, desempeñándose como médico de la familia real y adquiriendo gran renombre. Los servicios que brindó al emperador Alejandro I y a la emperatriz María Feodorovna le permitieron ganarse la confianza y el afecto de los mismos. En 1807 fue nombrado médico general del Departamento de Medicina Civil de Rusia y, tiempo más tarde, se le otorgo la Cruz de
San Vladimiro por su contribución a la lucha contra la epidemia de cólera que asoló las provincias del sudoeste ruso.

Crichton se destacó también por sus estudios de botánica, arqueología, zoología, geología y mineralogía, e integró diversas sociedades en Inglaterra y en Rusia, como la Academia Linneana y la Royal Geographical Society.

Durante sus múltiples viajes por Rusia, Noruega, Alemania, Reino Unido, Estados Unidos, Hungría, e incluso la lejana India, el médico inglés se dedicó a recolectar y ordenar una importante colección de minerales. Estas breves pinceladas sobre sus inclinaciones e intereses hablan claramente de un intelectual de espíritu inquieto dotado de una gran curiosidad por casi todos los aspectos de la cultura de su tiempo. En 1842 fue nombrado miembro de la Royal Academy de Gran Bretaña, y a partir de ese momento dedicó todo su tiempo a la Academia y a la práctica médica.

Alexander Crichton falleció el 4 de junio de 1856 en Kent, y sus restos fueron inhumados en el cementerio de Norwood.

Crichton no fue un alienista sino que, como Chiarugi y Reil, fue un médico generalista y publicó un solo tratado acerca de la locura, el cual ha pasado prácticamente desapercibido en la literatura médica, a excepción de los especialistas en historia y epistemología de la psiquiatría (Weiner, 1990); vale la pena consignar, además, que su obra recién fue accesible para los lectores hispanoparlantes en el año $2013^{7}$, ya que hasta ese momento solo se contaba con las ediciones inglesa, francesa y alemana.

Su texto An Inquiry into the nature and origin of Mental Derangement. Comprehending a concyse system of the physiology and pathology of the human mind and a history of the passions and their effects $(1798)^{8}$ cuenta con más de 300 páginas y está dividido en tres libros:

- Libro I: Investigación de las causas físicas del delirio (seis capítulos).

- Libro II: Historia natural de las facultades mentales y descripción de las enfermedades que las afectan o sistema conciso de la fisiología y patología de la mente humana (ocho capítulos).

- Libro III: Sobre las pasiones y sus efectos (seis capítulos).

En palabras del propio autor: «... en el primero investigo las causas físicas o corpóreas del delirio y 
otros trastornos de la mente. En el segundo se investigan los diversos cambios mórbidos a los cuales cada facultad de la mente humana está sujeta, ya sea por esfuerzo intenso o por actividad desmedida original o adquirida. Y en el último libro, trato las pasiones.» (Crichton, 2013, p. 194); y, diciendo más adelante: "me he concentrado demasiado quizás, en la fisiología de estas maravillosas afecciones de nuestra parte moral» (Crichton, 2013, p. 196).

En el Libro I analiza una serie de argumentos a favor de una visión materialista de la mente, en el Libro II discute con Joseph Priestley (1732-1804) y su teoría del monismo materialista aplicando los argumentos animistas y vitalistas adquiridos en su paso por Gotinga y en Libro III (la parte más conocida del texto que presentamos) aborda la cuestión de la fisiología de las pasiones (Charland, 2008).

Como lo ilustran las breves notas biográficas de Crichton $^{9}$, se puede afirmar que casi ningún aspecto de las ciencias de su época le era ajeno. Afanándose en todos sus trabajos con una gran pasión, fue justamente en ese terreno, el de las pasiones, en el que podemos registrar su contribución más significativa al acervo de lo que más tarde se constituiría en la moderna psiquiatría. Persona y tema se mezclan en este caso en forma imposible de separar, dando lugar entonces a una obra muy personal constituida con aportes provenientes de diversos contextos culturales, políticos y sociales.

En este artículo, y a manera de muestra de algunos de los diversos aspectos de una obra muy poco visi$\operatorname{tada}^{10}$, se desarrollarán ciertas temáticas del corpus teórico crichtoniano: algunas cuestiones referidas al método general que aplica, sus contribuciones a la semiología de la mente, al análisis del proceso de envejecimiento y a la función de la atención, para detenernos, finalmente, en su conceptuación del mundo de las pasiones y de la locura ${ }^{11}$. Ese recorrido tiene como objetivo acercar al lector interesado en estas cuestiones, en particular al hispanoparlante, una aproximación a esos aspectos de la obra de Alexander Crichton y subrayar su importancia, habitualmente soslayada, en relación con el nacimiento de la psiquiatría.

\section{EL MÉTODO GENERAL EMPLEADO POR CRICHTON}

En el prefacio de su Inquiry... Crichton presenta el método general de su obra; su importancia radica en que solo adentrándose en él es posible acceder a una comprensión acabada del desarrollo teórico desplegado por el autor. El interés por las Ilamadas enfermedades mentales, "oscura rama de la medicina»
(Crichton, 2013, p. 192), como Crichton las denomina, proviene de la constatación de que los intentos de echar luz sobre su naturaleza habían sido, según el autor, insuficientes y más bien escasos; opinión que deja bien establecida en uno de los párrafos iniciales afirmando: «... el trabajo que presentamos hoy al juicio del público es un intento de reducir, bajo ciertos principios, un número de hechos sueltos que abundan en los escritos de médicos, metafísicos y filósofos de diferentes edades y de diversos países [...] en general se confiesa que los intentos de echar luz sobre su naturaleza real han sido insuficientes $y$, por ende, no han sido exitosos» (Crichton, 2013, p. 192)., dejando entender que en las cuestiones que va a tratar no tiene pertinencia solo la medicina sino que la metafísica y la filosofía también tienen parte en su dilucidación. Este dato se debe tener muy en cuenta cuando se aborde, al final de este artículo, la problemática de las pasiones; aquí aparece de manera precoz la impronta germánica en su forma de encarar la presentación del texto, descartando de plano la posibilidad de que solo sea suficiente una mirada científica.

El autor plantea que el método que adoptó en todo el trabajo es el Análisis; entendiéndolo como el único criterio de prueba de la verdad, no solo en cuestiones de sentido externo sino también en objetos de razonamiento abstracto "ya que al realizar este tipo de investigación, cada componente o elemento constituyente debe ser examinado por separado; este es el modo más seguro de detectar errores, y como con él se aclaran todas las ideas complejas, en la medida que las partes individuales que las componen están claramente representadas en la mente, es el mejor modo de establecer resultados bien fundados» (Crichton, 2013, p. 193). Destacando, a continuación, que el éxito o el fracaso del Análisis dependerán del conocimiento previo de la persona que lo realiza, ya que requiere que quien emprenda la tarea esté familiarizado con la mente humana normal; debiendo el investigador aunar una visión clara y serena con la imparcialidad del historiador natural: «Obviamente se requiere que quien emprenda la tarea de examinar de este modo esta rama de la ciencia esté familiarizado con la mente humana en estado saludable, y que no solo sea capaz de abstraer su propia mente y ponerla fuera de sí mismo, por así decirlo, para poder examinar con la libertad y con la imparcialidad de un historiador natural, sino que también debe poder tener una visión clara y serena de cada causa que tiende a afectar las operaciones saludables de la mente y rastrear sus efectos. Debe ser capaz de volver a la infancia y ver como la mente esta modelada por la forma- 
ción recibida...» (Crichton, 2013, p. 193). Dora Weiner se detiene especialmente en este pasaje y lo relaciona, sagazmente, con las posteriores elaboraciones de Sigmund Freud (Weiner, 1990, p. 364).

Luego de desmembrar el todo, descomponiéndolo en sus partes o elementos para observar las causas, la naturaleza y los efectos de las partes constitutivas del fenómeno en estudio, el investigador, explica Crichton, debe proceder en forma inversa: «Cuando el trabajo del Análisis está completo, queda la parte más útil y difícil, la de aplicar el resultado o el principio general para explicar y ordenar los hechos individuales» (Crichton, 2013, p. 193).

Crichton relata que su interés por los trastornos de la mente humana se vieron reavivados a partir de recibir una publicación alemana, muy reputada en la época, que le fue recomendada por dos de sus mentores, los profesores Blumenbach y Arnemann ${ }^{12}$. Se trataba del Magazine zum Erfahrung Seelenkunde compuesta por ocho volúmenes y dirigida por Charles Moritz y Salomón Maimón ${ }^{13}$. Si bien, por un lado reconoce que la gran mayoría de los casos carecían de interés ya que eran enviados a los editores por distintas personas y no eran atrayentes para el profesional, por otra, el método desplegado en los mismos ejerció una profunda seducción en su espíritu debido a que en los mismos se describían casos de mentes alteradas contados en forma detallada y satisfactoria, como nunca antes había leído ( «... los alemanes prácticamente nos igualan en la debilidad por lo maravilloso, y debemos confesar que la Revista de Experiencia Psicológica contiene un amplio y rico surtido de materiales con los cuales este frágil deseo puede ser complacido. Las historias de sueños proféticos, sorprendentes inspiraciones y advertencias ocupan gran parte de la obra e, independientemente de ellas, la larga y frecuentemente tediosa relación de los sentimientos morales de los sordos, la historia de los crímenes, etc., en general no le interesan al médico») (Crichton, 2013, pp. 191-192).

La gran admiración que profesaba hacia la cultura alemana era el facilitador que le permitía aprovechar esos aportes, que en esos tiempos eran soslayados por los galenos franceses, quienes excepcionalmente hablaban alemán y estaban más concentrados en sus propias elaboraciones. Podría decirse que Crichton aportó el toque de observación clínica que faltaba a los casos de los filósofos-psicólogos alemanes, ya que estos no eran médicos. El corolario del método estaría dado por un intento básico, pero dirigido, de organizar y cuantificar las observaciones y es justamente en ese punto donde radicaría la diferencia entre el médico y el filósofo: "Es esto, efectivamente, lo que distingue al hombre de ciencia del mero intelectual» (Crichton, 2013, pp. 193).

Entre los referentes teóricos de Crichton, con quienes ora se inspira y ora discute discute, caben destacar dos grupos, uno alemán y el otro inglés, que lo influenciaron de manera concreta; en el primero encontramos a Johann Augustus Unzer (médico y psicólogo, sensualista, que tuvo un gran ascendiente sobre los médicos ingleses), a Hermann Ewald (con su trabajo «Sobre el corazón humano») y a Karl Schmidt (filósofo y teólogo, reputado pensador kantiano). En el segundo debemos subrayar a John Locke (pensamiento filosófico empirista, crítico al innatismo y al racionalismo para poner el eje en la experiencia concreta), a Thomas Reid (contemporáneo de David Hume, opuesto a Locke, influencio a la filosofía francesa de comienzos del siglo XIX) y Joseph Priestley (científico, teólogo, filósofo y educador, inventor del agua carbonatada y creador de la teoría del flogisto) (Stagnaro, 2013).

\section{LA MENTE, EL ENVEJECIMIENTO Y LA ATENCIÓN}

El Libro II, que será motivo de este apartado, intenta definir en su primera parte la naturaleza de la mente; aunque Crichton aclara desde el inicio que esa es una tarea ímproba ( .... como tratar de averiguar solo a través del pensamiento la naturaleza del Todopoderoso, si existió antes de los tiempos, o si Él mismo tuvo un comienzo») (Crichton, 2013, pp. 294).

Es importante remarcar que permanentemente, a lo largo de toda la obra, se hace mención a los límites del conocimiento humano, una apelación a una suerte de humildad epistemológica, bastante ausente en la mayoría de sus contemporáneos («... los límites de la razón humana están claramente marcados y pueden ser fácilmente discernidos por cada investigador, cualquiera sea el grado de entusiasmo con que lleva a cabo sus investigaciones, siempre y cuando su juicio no sea cautivado por la pasión del orgullo, o no este cargado del prejuicio extraño e inamovible acerca de que los poderes y la perfección del hombre no tienen límites») (Crichton, 2013, p. 294); la combinación entre el materialismo médico y la especulación filosófica alcanza su máxima expresión en este capítulo, debido al tenor de los temas que son abordados en el mismo.

Alertando contra aquellos que sacan conclusiones con demasiada rapidez, ya que la premura, dice Crichton, es enemiga del pensamiento; la transforma- 
ción de conjeturas en hipótesis, en un tema tan delicado como la naturaleza de la mente «... no debemos quedarnos satisfechos con las analogías más imprecisas que parecen cautivar a los hombres de gran imaginación y abstenernos de investigarla más a fondo» (Crichton, 2013, p. 295).

El autor discute con los investigadores de su época, sin privarse en ningún momento de criticarlos a través de una fina ironía «... algunos filósofos imaginaron que no había más de una clase de materia primitiva o elemental; y otros, al adoptar esta noción, parecen haberse regocijado de no estar ubicados en la escala de la naturaleza por encima de un pedazo de granito. Sin embargo, cada hecho que nos brinda la experiencia se opone a dicha conjetura [...] la mente tiene facultades y principios, pero esta es una circunstancia que hasta ahora ningún escritor de psicología ha observado suficientemente») (Crichton, 2013, p. 300).

La diferencia que establece entre facultad y principio de la mente es la que le permite avanzar en una interesante polémica con Priestley: «... las facultades modifican las impresiones sensoriales de varias maneras, proporcionándoles nuevas características y cualidades y convirtiéndolas en objetos de pensamiento y razonamiento [...] por otra parte los principios de la mente no modifican las impresiones sensoriales, que si son puestas en acción por ellos, y su acción se transfieren a las facultades...») (Crichton, 2013, p.301 ). La concepción de la ciencia que tenía Priestley concebía a la ciencia fue una parte integrante de su teología y, a lo largo de sus investigaciones, trató de fusionar el racionalismo de la Aufklarung con el teísmo cristiano; así como en sus obras de metafísica, se esforzó por combinar el teísmo con el materialismo y el determinismo (Tapper, 2002).

Partiendo de esta diferenciación Crichton avanza en la crítica de la obra del célebre científico, teólogo y filósofo inglés cuando sostiene enfáticamente que «... de todas las especies de materialismo, esa me parece ser la más absurda de todas, la cual se basa en la suposición de que el cerebro y la mente son una sola misma sustancia. Sin embargo, esta doctrina es aceptada por quien ha agregado hechos más útiles a la ciencia y más adornos a la filosofía que cualquier otro escritor moderno». La polémica adquiere ribetes muy personales, en tiempos donde las opiniones eran vertidas sin ninguna moderación: «A fin de demostrar el gran peligro de dicho razonamiento, o más bien de dichas aseveraciones (ya que no merecen llamarse razonamiento) solo tenemos que leer el trabajo del doctor Priestley al que se hace alusión y ver en que dilema cae por un modo similar de argumentación. De hecho él niega que la solidez y la extensión sean cualidades de la materia, y las define como simples centros de atracción y repulsión.») (Crichton, 2013, p. 301).

La conclusión a la que arriba Crichton es, que un cerebro enfermo ocasiona trastornos en la mente, entendiendo por cerebro enfermo a aquel afectado por las alteraciones de la irrigación que se producen a partir de «... los casos de fiebres e inflamaciones generales y locales de distintas clases». La mejoría siempre conlleva, según nuestro autor, una recuperación de las facultades mentales alteradas. Finalmente y como corolario de su teoría de la mente finaliza diciendo: «... la conclusión que podemos sacar a partir de estos hechos es en favor del materialismo, y es que la mente no es la sustancia del cerebro, sino la sangre o los fluidos segregados de ella en el cerebro» (Crichton, 2013, p. 303).

En las páginas dedicadas al proceso de envejecimiento cuestiona profundamente la teoría sostenida por la mayoría de los psicólogos de la época que afirmaba que a medida que nuestro cuerpo envejece y se debilita las facultades mentales decaen en forma irremediable; el desarrollo de algunas cuestiones en torno a la vejez se compadecen de la discusión acerca de la materialidad del alma que se viene comentando en este apartado. En efecto, en palabras de Crichton: «... si se puede probar que la mayoría de las personas que ejercitan debidamente la memoria, la imaginación, el juicio y todas las demás facultades mentales, mejoran gradualmente su mente mientras su cuerpo va decayendo, es seguramente más que una prueba supuesta el hecho de que los elementos del alma (si se me permite la expresión) son muy distintos de los materiales que componen el cuerpo» (Crichton, 2013, p. 304).

Para apoyar sus ideas cita al conocido Ensayo sobre la Ancianidad escrito por Marco Tulio Cicerón ${ }^{14}$, donde se pueden encontrar numerosos ejemplos de hombres cuyo intelecto se mantiene totalmente vigoroso luego de que sus fuerzas físicas han desaparecido casi por completo («Platón muere a los ochenta y un años mientras trabajaba en la elaboración de una obra. Isocrates finalizó su Panathenaicus a los noventa y cuatro años de edad y su maestro Leontinus Gorgias trabajaba igual que cualquier hombre a la edad de ciento siete años») (Crichton, 2013, p. 305).

Para dar más fuerza aun y potenciar sus argumentos selecciona algunos ejemplos más contemporáneos de ancianos lúcidos que grafican su pensamiento («... 
Lord Mansfield, el Dr. Samuel Johnson, Voltaire, Erasmo Darwin, autor de Zoonomia y The Botanic Garden ¿no son pruebas directas de que la mente sigue mejorando luego de que el cuerpo comienza a decaer? Sir Isaac Newton, cuando estaba cerca de cumplir ochenta años resolvió el famosos problema de las trayectorias, el cual había enviado Leibniz a este país con el objeto de hacer pensar al primer matemático de la época») (Crichton, 2013, p.305).

Con estos ejemplos de envejecimientos exitosos el autor intenta demostrar que «Para ser justos es necesario mencionar que, a pesar de que se puede probar que los fenómenos de la mente son muy distintos de los que parecen pertenecer al cerebro y a los nervios, no aclaran la duda en cuanto a la materialidad o inmaterialidad del alma ya que, a pesar de ser esencialmente diferente al cerebro, aun así puede ser materia» (Crichton, 2013, p. 306).

A lo largo de toda la obra Crichton procura separar aquellas cuestiones que podríamos llamar científicas, que se sostienen por argumentos, de aquellas otras que el mismo denomina creencias, totalmente subsumidas a cuestiones inmanentes al mundo de la fe, alejado del mundo de las ciencias ( $" . .$. aquellos que piensan que no es materia, no basan su opinión en argumentos sino en creencias. La evidencia de nuestros sentidos, la fuente principal del conocimiento, no nos enseña ningún hecho similar a esto. La doctrina sobre las inmaterialidades es, por lo tanto, un tema de fe y no de razón»). En este pasaje se advierte que el énfasis esta puesto en que los sentidos son la clave de todo intelección y de todo saber, y nada puede haber que vaya más allá de los mismos ( «... esta suposición lo lleva a investigar qué es eso llamado materia, y pronto descubre, si no es corrompido por ninguna hipótesis, que no puede obtener desde la experiencia una idea clara de su naturaleza real») (Crichton, 2013, p.307). Las ideas que Crichton esboza acerca del fenómeno del envejecimiento no fueron rescatadas por los científicos que lo sucedieron, quedando más bien como especulaciones filosóficas (a la manera del malogrado Cicerón), permaneciendo sumergido dicho proceso en una continuidad de prejuicio que llega aún hasta nuestros días.

Para continuar, cabe una mirada al capítulo que se ocupa de la atención y sus enfermedades; en principio Crichton la reconoce como una de las facultades de la mente. Para comenzar el autor formula la atractiva hipótesis según la cual plantea las diferencias cualitativas en cuanto a la capacidad de prestar atención por parte de los diferentes individuos; y se detiene, especialmente, en la predisposición que manifiestan algunas personas a experimentar ciertas pasiones o emociones (un anticipo del Libro III) y no otras: «... un hecho fácilmente comprobable por la experiencia diaria es que algunos hombres, debido a su organización o constitución corporal, son más propensos a ciertas emociones y pasiones que a otras como, por ejemplo, las emociones violentas de la ira y sus modificaciones, las emociones del temor, el deseo sexual y sus modificaciones, etc.» (Crichton, 2013, p. 312).

Propone que ciertas direcciones de la atención, más allá de que «... a pesar de que los deseos y emociones a las cuales ciertos individuos están sujetos por la conformación original de los nervios, y que le otorgan, necesariamente, una preferencia a la facultad de la atención, especialmente entre los hombres más ignorantes y salvajes, y los llevan a involucrarse con los objetos que provocan estas afectaciones», pueden ser modificadas con la enseñanza y el aprendizaje aplicados con sabiduría durante la primera infancia. El enfoque siempre es equilibrado, ya que siempre al bagaje biológico le suma los elementos provenientes de la vida de la persona ( $" . .$. nuestro modo de educación, nuestros pasatiempos, nuestra profesión y por otras relaciones cotidianas con el mundo externo») (Crichton, 2013, p. 312).

Crichton describe, luego, lo que el denomina «enfermedad de la atención» y la caracteriza de la siguiente manera: «... surge en forma casual [...] es inducida también por problemas estomacales, por la clorosis y la hidrofobia [...] cada impresión parece generar agitación en la persona y un grado antinatural de inquietud mental [...] Personas yendo y viniendo en un lugar, un ruido, una mesa que se mueve, una puerta que se cierra en forma repentina, el ligero exceso de calor o frío, mucha luz o muy poca luz, son causas que destruyen la atención constante en dichos pacientes, ya que es cautivada fácilmente por cada impresión») (Crichton, 2013, p. 316).

El autor dedica varias páginas al tema de la educación, siendo muy crítico con la misma ( $" . .$. ¿no es frecuente que un niño sea sometido a la fatigosa tarea de cargar su memoria con meras palabras y que las facultades activas de su alma se tornen inertes...?»), llegándola a acusar de haber malogrado no pocos talentos que terminaron perdiéndose en «la fatiga mental o adquiriendo gran desagrado por la instrucción porque los estímulos adecuados que atraen su atención no se han descubierto a tiempo» (Crichton, 2013, p.314). La formación, la enseñanza y el aprendizaje eran una de las obsesiones de Crichton y de su época, y aun en 
una sección del libro como ésta, dedicada a aspectos semiológicos y teóricos, abundan las reflexiones consagradas a la instrucción y sus consecuencias. Es en esta línea que sostiene que en muy contadas ocasiones se tienen en cuenta los aspectos singulares de los individuos a la hora de definir su formación: «Desafortunadamente, sucede que el tratamiento mental de los niños, no solo en los colegios y en las academias, sino también en los hogares es, en general, el mismo para todos, y algo similar sucede con las niñas. Pocas veces se tienen en cuenta las peculiares idiosincrasias o tendencias individuales» (Crichton, 2013, p.318).

Las descripciones que siguen están relacionadas con aquellos infantes que «requieren distintos objetos de estudio para poder llamar su atención en forma suficiente y ejercitar sus mentes en forma adecuadas». Dice Crichton: «Todo profesor debe haber observado que para muchos las monótonas y difíciles gramáticas del latín y del griego son tan desagradables que ni siquiera el miedo a la varilla o el trato indulgente genera que presten atención. Si se determina que un niño de esta disposición no es deficiente en cuanto a la comprensión natural, ¿Por qué se pierden tantos años en infructuosos intentos que seguramente son fastidiosos para el tutor y perjudiciales para los niños? ¿Un médico sabio consideraría que un tipo de alimentación seria apropiada para todo tipo de complexión? Se puede ver que en general los niños con voluntad se interesan fácilmente por otras ramas del estudio. Si ese es el caso, la inclinación natural de la mente no debería ser frustrada a la fuerza ni abandonada» (Crichton, 2013, p. 318-319) ${ }^{15}$.

En este capítulo así como en los anteriores y en los subsiguientes los nervios y sus conexiones ocupan un lugar central en la obra crichtoniana, ya sea por déficit o por exceso están siempre involucrados en las diversas afecciones descriptas ( Se ha comentado que la debilidad y el letargo del cuerpo son causas que debilitan la atención debido a que los nervios de dichas personas no transmiten las impresiones recibidas con el grado necesario de intensidad y claridad») (Crichton, 2013, p.319).

\section{CRICHTON, PINEL, ESQUIROL Y LAS PASIONES}

El Libro III trata sobre las pasiones y sus efectos, y es, sin duda, la parte más visitada y conocida de la obra de nuestro autor a pesar de que este fenómeno dista mucho de alcanzar la difusión que merece; este hecho estaría relacionado con que el énfasis puesto por el médico escocés en el estudio de las pasiones aparentemente influenció el pensamien- to tanto de Pinel como de Esquirol (Weiner, 1999; Stagnaro, 2013); para Crichton la clave residía en una prolongada observación de los sentimientos de los pacientes, expresados en palabras, gestos o actitudes ya que esta estrategia era la única que permitía arribar a un diagnóstico, manteniéndose alejado de cualquier tipo de especulación. En este punto podemos decir que los caminos del escocés y del francés se cruzan, ya que ambos proponían poner el foco en el paciente. De todas formas Pinel va mas allá debido a la propuesta y a la sistematización del tratamiento moral, mientras que con su Inquiry... Crichton realiza un aporte único y aislado al nacimiento de la especialidad. De todas formas podemos decir que al intentar hacer desaparecer todo atisbo de juicio o de moralización se da un paso trascendental en la transformación del loco en paciente.

Los capítulos sobre la alegría, la tristeza, la melancolía, el miedo, la ira y el amor, pasajes clave para entender la concepción del autor acerca de las pasiones, constituyen un intento de desarrollar una psicopatología puramente fisiológica, y totalmente independiente de consideraciones éticas o morales. En sus propias palabras: «... los moralistas y los metafísicos han escrito mucho, pero se han limitado solamente al ámbito moral y metafísico; las investigaciones que han realizado no son útiles para el médico [...] las pasiones deben considerarse desde un punto de vista médico y deben examinarse con el ojo de un historiador natural y la imparcialidad de un filósofo [...] no nos concierne si las pasiones se consideran afecciones naturales o antinaturales o morales o inmorales [...] son fenómenos cuyas causas naturales deben ser investigadas» (Crichton 2013, p. 415).

Pinel conocía los postulados de Crichton ${ }^{16}$; esto queda demostrado en la introducción de la primera edición del Traité ${ }^{17}$, en 1800 (Pinel, 1988, pp. 37-56), donde el francés sostiene que el libro de Crichton es, en líneas generales, un trabajo profundo que propone fundamentalmente nuevas observaciones basadas en la moderna fisiología de la época, dedicando más de veinte páginas a desarrollar sus ideas al respecto del texto del inglés; Berrios sostiene que Pinel estaba profundamente impresionado por el hecho de que Crichton usara el concepto de pasión en su Libro III en forma fisiológica, no metafísica («... sin ningún tipo de referencias a la moralidad o a la inmoralidad») (Berrios, 2006, p. 476; Weiner, 1999).

Charland argumenta que lo que más impresiono a Pinel y a Esquirol fue el rastreo riguroso de la psicopatología de las pasiones, retomado por estos autores 
en sus propias elaboraciones acerca de cuál era el lugar de la pasión en la enfermedad mental; asimismo sostiene que la mirada de Crichton era puramente fisiológica, soslayando cualquier tipo de aporte filosófico, ético y moral, a pesar de tomarlos en cuenta (Charland, 2008).

Berrios problematiza en detalle la cuestión de las «nuevas observaciones», planteando en forma enfática que en el libro de Crichton no existe ninguna nueva observación (en el sentido de aporte personal u original), ya que todos los casos presentados fueron tomados prestados de la fisiología de Unzer y de la Revista de Experiencia Psicológica. Nuestro autor comenzó a recolectar material para su libro a los treinta años de edad, hasta entonces su experiencia en diagnósticos de locura o en manejo de este tipo de pacientes era inexistente. Berrios concluye (diferenciándose de Weiner) en que las influencias de Crichton sobre los franceses no quedan del todo aclaradas. Pareciera que el escocés impresionó más a Esquirol que a Pinel, ya que el primero dispuso de tiempo para leer su obra antes de publicar su tesis, mientras que si bien Pinel conocía el trabajo de Crichton previamente a la publicación de la primera edición de su tratado, las ideas principales sobre las pasiones ya habían germinado en su mente con anterioridad a la redacción; y en la segunda edición del Tratado le dedica solamente dos páginas de la introducción, reduciendo drásticamente el reconocimiento y la valoración del trabajo del inglés (Berrios, 2006; Charland, 2008).

La tesis de Esquirol Des passions... publicada en 1805 , nos remite desde su título a la obra de Crichton, que sin duda fue leído y estudiado detenidamente por el autor francés (Esquirol, 1805). Un planteo que subyace a estos trabajos es el uso que se le podría llegar a dar a las pasiones en el tratamiento de los pacientes; Weiner plantea que Pinel y Crichton proponían que si el paciente comprendía sus pasiones erradas podía llegar a cambiar sus conductas, mientras que Esquirol proponía usar las pasiones como un tratamiento de shock que permitiera que el paciente recuperara la razón (Weiner, 1990).

Es tentador pensar a Crichton, siguiendo la opinión de Weiner, como el eslabón perdido entre la clínica francesa y la alemana ya que como se ha consignado antes en este trabajo, las vicisitudes de su formación le permitieron sintetizar y aplicar los aportes obtenidos en un modelo de pensamiento original que posteriormente no fue retomado por otros autores, a diferencia de la saga iniciada, continuada y profundizada por Pinel y Esquirol ${ }^{18}$.

De todas formas, consideramos que las relaciones, similitudes y diferencias entre estos autores están lejos de haber sido investigadas a fondo, creemos que es necesario avanzar aún más en la lectura crítica de Crichton, consideramos que sería adecuado intentar una sistematización (cosa que si se ha hecho con la obra de Pinel) debido a que existen numerosos matices que no han sido considerados; tal vez quien más se acercó a ello fue Charland, pero su trabajo se centra en el Libro III acerca de las pasiones (Charland, 2008), quedando el resto de la obra fuera de su foco de análisis.

\section{NOTAS}

1 Alexander Wood (1817-1884) oriundo de Edimburgo, como Crichton, es recordado por ser el inventor de la jeringa hipodérmica, que perfeccionó y popularizó el francés Charles Gabriel Pravaz (1791-1853).

2 William Fordyce (1724-1792) médico escocés, miembro del Royal College of Physicians y de la Royal Society, conocido en su época por sus estudios y publicaciones acerca de las fiebres y el uso medicinal del ruibarbo.

3 Dora Weiner señala la frase de Crichton "... tuve el honor de conocer personalmente" [a Pinel] que el inglés menciona en la página 180 de sus Commentaries on some doctrines of a dangerous tendency in medicine, Londres, 1842 (Weiner, 1999, p. 300).

4 Maximilian Stoll (1742-1787) médico austriaco, profesor de la universidad de Viena en la que sucedió a Anton de Haen (1704-1776) como director de la clínica adjunta. Stoll, quien se destacó como epidemiólogo y creador de una nosología inspirada en la de Thomas Sydenham (1624-1689), trabajó en estrecha colaboración con otros miembros de la célebre Escuela de Viena como Leopold Auenbrugger (1722-1809) (junto al que desarrolló la metodología de la percusión), Gerard van Swieten (17001772) y Anton von Störck (1731-1803), y tuvo entre sus alumnos a Franz Joseph Gall (1758-1828).

5 Philipp Friedrich Theodor Meckel (1755-1803), miembro de una familia de médicos anatomistas muy renombrados, accedió en 1777 al cargo de profesor titular de la cátedra de anatomía, cirugía y obstetricia de la universidad de Halle. 
6 La personalidad de Blumenbach (antropólogo, médico y psicólogo, creador de la llamada antropología física que aplicaba conocimientos derivados de la anatomía comparada), parece haber ejercido un efecto definitivo en nuestro autor: gran amante de los viajes y dotado de una mirada humanística fuera de lo común, llego incluso a atesorar una colección de calaveras muy conocida en su tiempo (Berrios, 2006).

7 Crichton, Alexander "Investigación sobre la naturaleza y el origen del trastorno mental", 1era. edición en castellano, Buenos Aires: Polemos, 2013. El volumen que contiene esa obra lleva como título genérico "Los prolegómenos del tratamiento moral”, y reúne, además del libro de Crichton, textos de William Battie, John Monro, Jean Colombier y François Doublet y Joseph Daquin, y cuenta, además, con una introducción con notas biográficas de los autores de Juan Carlos Stagnaro, compilador de la obra. Todas las referencias a la obra de Crichton en este artículo corresponden a esa versión en castellano.

8 Crichton se reivindica como el primer autor de Inglaterra (curiosamente aunque los menciona después en su texto no les reconoce ese estatus a William Battie y a John Monro, por ejemplo) que "ha escrito en detalle sobre las Enfermedades Mentales", a excepción de Thomas Arnold (17421816) de Leicester, a quien menciona como antecedente, al igual que al francés Jean-François Dufour autor del Essai sur les opérations de l'entendement humain et sur les maladies qui les dérangent, en 1770, y al alemán Johann August Unzer (1727-1799) en el suyo (Crichton, 1798 [2013] p. 189 y 190). Arnold había publicado, en 1782, Observations on the Nature, Kinds, Causes and Prevention of Insanity y Dufour había hecho lo propio con su Essai sur les opérations de l'entendement humain et sur les maladies qui les dérangent, en 1770. Nada dice de otros autores que lo precedieron como Vincenzo Chiarugi, ni Joseph Daquin, ni del informe de Jean Doublet y François Colombier de 1787.

9 Para una información biográfica más detallada véase Tansey E. (1984) Appleby, J. y Appleby RH (1999).

10 Existen tres grandes excepciones de consulta indispensable y de las cuales el presente texto es deudor: Weiner D (1990) Berrios G (2006) y Charland L (2008).

11 En este escrito no se incluye la elaboración que Crichton realiza en torno a la cuestión de los delirios (libro primero), debido a que la complejidad y la longitud del tema excede el programa propuesto para el mismo. Solamente cabe informar aquí, a modo de brevísimo resumen que el autor indaga conceptos como la irritabilidad y sus leyes, la sensación y los sentidos externos, la conciencia del yo, las sensaciones de dolor y placer corporal y los delirios (Crichton, 1798 [2013], pp 203-291). El tema del delirio es un punto fundamental, que Pinel, quien tradujo ese capítulo del libro de Crichton al francés en el primer volumen (1798-99) del Recueil périodique de littérature médicale étrangère (Berrios, 1996; Wiener, 1999), recoge permitiéndose disentir con el médico escocés, tanto por el hecho de que su teorización se hace a partir de un solo caso, cuanto porque no toma en cuenta a las emociones (Weiner, 1990). Es cierto, por otro lado, que Pinel contaba con una experiencia clínica sobre las enfermedades mentales que Crichton no tenía (Weiner, 1999).

12 Justus Arnemann (1763-1807), profesor de medicina y cirugía en la universidad de Gotinga.

13 Karl Phillipp Moritz (1757-1793) era un escritor, amigo dilecto de Goethe, además de editor y crítico de arte; mientras que Salomón Maimón (1752-1800) era un distinguido filósofo judío y experto en cuestiones cabalísticas (Berrios 2006; Stagnaro, 2013).

14 Uno de los pasajes de ese texto pudo inspirar estas nociones en Crichton; en el mismo se presenta un diálogo entre tres personajes históricos: Catón el viejo, entonces de 84 años, y dos jóvenes: Escipión menor, hijo del famoso Paulo Emilio, y su amigo Cayo Lelio. Éstos se asombran de que el estadista hubiera alcanzado una edad muy madura y que se encontrara en plenas condiciones físicas y mentales. Catón les da sus razones, a la par que desbarata cuatro motivos según los cuales la vejez resulta miserable: $1^{\text {o) }}$ La vejez aparta de las actividades; sí, de las del cuerpo, pero las grandes cosas no se hacen con la fuerza, sino con el consejo, la autoridad y la opinión; 2) Hace perder la fuerza física; eso es indudable, pero ese debilitamiento puede ser morigerado mediante ejercicios corporales, alimentación adecuada y espíritu altivo; 3 ) La edad provecta impide concretar ciertos placeres; es cierto, pero disminuye también el deseo de experimentarlos e incluso permite disfrutarlos aunque se los vea desde lejos; 4ㅇ) Está cerca de la muerte. Es verdad, pero ya que no somos inmortales debemos saber aceptar nuestra partida con serenidad, sólo así la vida puede ser más llevadera ( $\mathrm{Ci}$ cerón Marco Tulio, 2001).

15 Esta última aseveración y algunas de las vertidas con anterioridad han sido tomadas por varios autores contemporáneos que han estudiado la historia del constructo Trastorno por Déficit de Atención con hiperactividad para atribuir la primera descripción de dicho síndrome a Alexander Crichton. Se pueden citar al menos dos trabajos, el primero de ellos de Palmer y Finger publicado en el año 2001 en Child Psychology and Psychiatry Review; en el mismo estos autores narran el trabajo de Crichton que estamos reseñando para concluir diciendo que la descripción hecha por nuestros autor antedata en más de cien años a la realizada por el pediatra George Still. En el otro trabajo, escrito por Lange, Reichl, Lange, Tucha y Tucha en el 2010 y publicado en Attention Deficit and Hiperactivity Disorders, que cita como fuentes biográficas principales tanto a Palmer y Finger como a Tansey; textualmente los autores plantean que "The first example of a disorder that appears to be similar to ADHD was given by Sir Alexander Crichton in 1798", para continuar diciendo "In this short description of the first alteration of attention, Crichton gives several indications that he was depicting the same disorder as defined in the current DSMIV-TR criteria of ADHD", y, por ultimo, "However, Crichton's descriptions provide some evidence for the existence of ADHD at the end of the eighteenth century". 
Estas aseveraciones nos parecen arriesgadas debido a que, entre otras cosas, fueron formuladas a partir de una serie de observaciones mínimas, donde los casos ni siquiera se hallan registrados; por otro lado Crichton no continuó publicando ni registrando sus reflexiones y solo se cuenta con el capítulo de semiología de la atención en el Libro II de su única obra dedicada a la psiquiatría, donde ni siquiera encontramos el historial de un paciente sino solo observaciones generales en torno a la cuestión de la atención patológica en la niñez.

\section{BIBLIOGRAFÍA}

Appleby, Robert (1999), "Sir Alexander Crichton, F.R.S. (17631856), imperial Russian physician at large", Notes and Records of the Royal Society of London, 53, pp. 219-230.

Appleby, John (2004), "Sir Alexander Crichton", Oxford Dictionary of National Biography, Oxford University Press, [online] available in: http://www.oxforddnb.com, [consultado 10 de octubre de 2014]

Berrios, G. (1996), The history of mental symptoms. Descriptive psychopathology since the Nineteenth Century. Cambridge, Cambridge University Press.

Berrios, G (2006), “Mind in general, by Sir Alexander Crichton with and introduction by G. E. Berrios", History of Psychiatry, 17 (4), pp. 469-497.

Ciceron, Marco Tulio (2001), De Senectute. Madrid, Editorial Triacastela.

Crichton, A. (2013), "Investigacion sobre la naturaleza y el origen del trastorno mental". En Battie, William; Monro, John; Colombier, Jean; Doublet, Francois; Daquin, Joseph; Crichton, Alexander, Los prolegómenos del tratamiento moral. Buenos Aires: Polemos, pp. 182-517.

Charland, Louis (2008), "Alexander Crichton on the psychopathology of the passions", History of Psychiatry, 19 (3), pp. 275-296.

Esquirol, Jean-Étienne Dominique (1805), Des passions considérées comme causes, symptômes et moyens curatifs de I'aliénation mentale, Thèse de médecine de Paris $n^{\circ} 574$, Paris, Didot Jeune.

Lange, Klaus; Susan Reichl; Katharina Lange, et al. (2010), "The history of attention deficit hyperactivity disorder", Attention Deficit Hiperactivity Disorder, 2 (4), pp.241-255.
16 El texto de Crichton es de 1798, Pinel traduce el capítulo sobre los delirios de ese libro en 1798-1799 (ver nota 11), es decir, casi dos años antes de editar su Tratado.

17 Pinel, Philippe (1800) Traité medico-philosophique sur I'aliénation mentale ou la manie, Paris, Richard, Gaille et Ravier. Se utiliza la versión en castellano Pinel, 1988.

18 Vale la pena consignar que en Alemania fue traducida la obra de Crichton antes que la obra de Pinel (Weiner, 1990).

Palmer, Erica and Stanley Finger (2001), "An Early Description of ADHD (Inattentive Subtype): Dr. Alexander Crichton and 'Mental Restlessness' (1798)', Children Psychology and Psychiatry Review, pp. 66-73.

Pinel, Philippe (1988) Tratado médico-filosófico de la enagenación mental o manía. Madrid: Nieva).

Stagnaro, Juan Carlos (2013), "Apuntes biograficos”. En: Battie, William; Monro, John; Colombier, Jean; Doublet, Francois; Daquin, Joseph; Crichton, Alexander, Los prolegómenos del tratamiento moral, Buenos Aires. Editorial Polemos, pp. IXXXIII.

Tansey, Edward (1984), "The life and works of Sir Alexander Crichton, F.R.S. (1763-1856): a Scottish physician to the Imperial Russian Court", Notes and records of the Royal Society of London, 38, pp. 241-259.

Tapper, A. (2002), “Joseph Priestley.” In: Philip B. Dematteis and Peter S. Fosl, (eds.), British Philosophers 1500-1799 in Dictionary of Literary Biography, Detroit, Gale Group.

Unzer, Johann August (1771), Erste Gründe einer Physiologie der eigentlichen thierischen Natur thierischer. Leipzig, Körper.

Weiner, Dora (1990), "Mind and body in the clinic: Philippe Pinel, Alexander Crichton, Dominique Esquirol, and the birth of psychiatry". En: Rousseau, Gerald (ed.), The languages of Pyche: mind and body in enlightment thought, Berkeley, University of California Press, pp. 331-391.

Weiner, Dora (1999), Comprendre et soigner. Philippe Pinel (1745-1826). La médecine de l'esprit. Paris, Fayard. 